Check for updates

Cite this: RSC Adv., 2017, 7, 40020

\title{
Synthesis of zinc-based acrylate copolymers and their marine antifouling application $\dagger$
}

\author{
Rongrong Chen, ${ }^{\text {ab }}$ Yakun Li, ${ }^{a}$ Liang Tang, ${ }^{a}$ Haocheng Yang, ${ }^{a}$ Zetong Lu, ${ }^{c}$ Jun Wang, ${ }^{\text {ab }}$ \\ Lianhe Liu*ad and Kazunobu Takahashi (DD *ab
}

Marine fouling organisms have caused inconvenience to humans for a long time owing to their high vitality and great destructiveness. Self-polishing antifouling coatings are considered to be among the most effective antifouling technologies. In this study, zinc-based acrylate copolymers (ZnPs) were designed and synthesized using a bifunctional zinc acrylate monomer (ZnM) as a new self-polishing monomer, and three acrylate monomers (namely, methyl methacrylate, ethyl acrylate and 2-methoxyethyl acrylate) were used as comonomers. ZnPs that contained the new ZnM were characterized by Fourier transform infrared spectroscopy, proton nuclear magnetic resonance spectroscopy and gel permeation chromatography. Different antifouling coatings were prepared using the previously mentioned $\mathrm{ZnPs}$ as the matrix material, and their erosion properties were investigated using a lab rotor test. A field test of the prepared coatings at various geographical locations showed their excellent antifouling performance as they inhibited the settlement of barnacles in both the South China Sea for 9 months and in the Yellow Sea for at least 15 months. The results of this study highlight that the biocidal ZnP-based coatings are highly promising candidates for marine antifouling applications.

Received 30th April 2017

Accepted 31st July 2017

DOI: $10.1039 / \mathrm{c} 7 \mathrm{ra0} 4840 \mathrm{~h}$

rsc.li/rsc-advances
Self-polishing antifouling coatings that contain a hydrolyzable resin, antifouling agents, and other additives have been used as highly effective antifouling coatings and are preferred in modern antifouling techniques. ${ }^{\mathbf{1 2 - 1 4}}$ Owing to environmental protection requirements, tin-free self-polishing antifouling coatings, which contain copper- or zinc-based acrylate copolymers, were developed as alternative antifouling coatings. They achieved the same performance as organic tin-based antifouling coatings without a significant increase in cost. ${ }^{12,15-17}$ With self-polishing coatings, the removal of the outer surface of the coating enables the mechanical cleaning of ship hulls and reduces their roughness. The prevention of fouling primarily relies on the release of active or biocidal ingredients that are contained in the paint formulation, and this release is closely related to the erosion rate of the paint sample. To ensure long-lasting antifouling performance, the efficient release of biocides must be prolonged as much as possible by controlling the erosion rates of antifouling coatings in accordance with the sailing conditions of the ship. Self-polishing copolymers facilitate the continuous renewal of the surface and the release of antifoulants via a hydrolysis reaction or an ion exchange reaction \footnotetext{
with seawater. In previous studies, a series of ZnPs were determined to be suitable for marine antifouling applications. ${ }^{18,19}$ The influence of the resin composition such as the molecular weight, content of $\mathrm{ZnM}$ and hydrophilicity of the comonomers was investigated, and a mechanism of biocide release and antifouling performance was proposed. ${ }^{20,21}$ In addition, the distribution of hydrolysable monomers within the polymer chains appears to affect leaching-out patterns in seawater. Commercial self-

Key Laboratory of Superlight Material and Surface Technology, Ministry of Education Harbin Engineering University, Harbin 150001, China. E-mail: liulianhe@hrbeu.edu. cn; kazunobu@163.com

${ }^{b}$ Institute of Advanced Marine Materials, College of Materials Science and Chemical Engineering, Harbin Engineering University, Harbin 150001, China

${ }^{c}$ College of Mechanical Engineering, Heilongjiang University of Science and Technology, Harbin 150022, China

${ }^{d}$ Qingdao Advanced Marine Material Technology Co., Ltd., Qingdao 266100, China

$\dagger$ Electronic supplementary information (ESI) available. See DOI: $10.1039 / \mathrm{c} 7 \mathrm{ra04840h}$
} 
polishing resins containing zinc methacrylate monomer have been employed as a good matrix for self-polishing coatings. However, the higher glass transition temperature of these resins tended to restrict the fluidity of the resin, which negatively affected the storability and decreased the stability of the self-polishing resin..$^{22}$ Paints based on these resins became less flexible as the zinc acrylate content increased. Therefore, their physical properties need to be improved. Although the polishing rate of paints is substantially affected by the leaching out of polymers, the composition and structure of ZnPs must be optimized.

Therefore, the control of the leaching out of polymers by varying the hydrolysis properties and paint formulation is required to ensure long-lasting antifouling performance. In this study, ZnPs were designed and synthesized using a bifunctional zinc acrylate monomer (ZnM) as a new self-polishing monomer. Three acrylate monomers, namely, methyl methacrylate (MMA), 2-methoxyethyl acrylate (2MEA) and ethyl acrylate (EA), were chosen to impart suitable properties to the copolymer resin for use as a surface coating. The molar ratio of zinc acrylate monomer to the comonomers that participated in the hydrolysis of the self-polishing coating was varied, and the structure and molecular weight of the polymer were investigated. Then, the erosion performance was investigated by dynamic immersion in simulated seawater. The antifouling performance was examined by a raft test. The relationship between the chemical structure of the corresponding polymers and their erosion resistance was also studied. Field experiments were performed to assess their efficiency in inhibiting the settlement of macrofoulers such as algae, mussels, hydroids and barnacles in situ. The antifouling performance of the coppertype coatings was investigated at two immersion sites with variations in fouling pressure (i.e., the South China Sea in the subtropical zone and the Yellow Sea in the middle temperate zone).

\section{Experimental}

\subsection{Materials}

Acrylic acid (AA, AR), methacrylic acid (MAA, AR), ethyl acrylate (EA, AR), methyl methacrylate (MMA, AR), zinc oxide (ZnO, AR), propylene glycol methyl ether (PGM, AR) and xylene (AR) were all purchased from Tianjin Zhiyuan Chemical Reagent Co., Ltd., China. 2,2'-Azobis(isobutyronitrile) (AIBN, AR), azobis(2methylbutyronitrile) (AMBN, high purity) and benzoyl peroxide (BPO, AR) were obtained from Tianjin Guangfu Chemical Reagent Co., Shanghai Yuanye Bio-Technology Co. and Shanghai Shanpu Chemical Co., Ltd., respectively. 2-Methoxyethyl acrylate (2MEA, >98\%) and Nofmer MSD (methylstyrene dimer: chain transfer agent) were purchased from Aladdin Chemistry Co. (Shanghai, China) and Adamas Reagent Co. (Shanghai, China), respectively. Deionized water was purified with a Milli-Q Plus system (Millipore, Schwalbach, Germany). All monomers were used without further purification.

\subsection{Synthesis of ZnPs}

2.2.1 Synthesis of bifunctional ZnM. Firstly, 85.4 parts PGM and 40.7 parts $\mathrm{ZnO}$ were charged into a four-neck flask equipped with a cooling tube, thermometer, dropping funnel and stirrer, and the mixture was heated to $75^{\circ} \mathrm{C}$. Then, a mixture consisting of 43.1 parts MAA, 36.1 parts AA and 5 parts water was added dropwise over 3 hours at a constant speed. After the addition was completed, the reaction solution changed from opaque to transparent. Then, the solution was stirred for 2 hours and 36 parts PGM was added to give a transparent mixture in which the zinccontaining monomer (namely, ZnM) was dissolved. The solid content was approximately $44.8 \%$.

2.2.2 Synthesis of ZnPs. A mixture of PGM, 57.0 parts xylene, and 4.0 parts EA was heated to $100{ }^{\circ} \mathrm{C}$ under agitation. Next, a transparent mixture of 5.0 parts MMA, EA, 2MEA, ZnM, 10.0 parts xylene, 1.0 part chain transfer agent (MSD), 2.5 parts AIBN and 7.0 parts AMBN was added dropwise over 6 hours at a constant speed. After the addition was completed, 0.5 parts BPO and 7.0 parts xylene were added dropwise over 30 minutes and the mixture was stirred for an additional 1.5 hours, followed by the addition of 6.6 parts xylene to give a slightly turbid pale yellow metal-containing resin (namely, $\mathrm{ZnP}$ ). The quantities of the main monomers, including MMA, EA, 2MEA and ZnM, are shown in Table 1. The initial quantity of PGM was adjusted according to the amount of $\mathrm{ZnM}$ solution in the formulation to allow all ZnPs to have a heated residue content of $48.2 \%$. ZnPs with various monomer compositions were prepared to investigate the effects of the ZnM content and the proportions of EA and MMA as hydrophobic monomers on their antifouling performance. The molecular compositions and characterization of the ZnPs and the definitions of the sample abbreviations are given in Table 1. The $M_{\mathrm{n}}$ and polydispersity index (PDI) of the ZnPs, which were determined by gel permeation chromatography (GPC), were approximately $2.3 \times 10^{3} \mathrm{~g} \mathrm{~mol}^{-1}$ and $1.85-2.18$, respectively.

\subsection{Antifouling paint formulation}

The prepared polymers were premixed with cuprous oxide $\left(\mathrm{Cu}_{2} \mathrm{O}\right.$, 98\%, Shandong Heze Chenxu Chemical Co., Ltd.) and copper pyrithione (CuPT, 97\%, Yixing Liaoyuan Chemical Co., Ltd.). These were the main biocides in the formulation, together with other ingredients, as shown in Table 2. These ingredients were processed in a paint conditioner for approximately 30 minutes. Then, the paints were filtered through a sifter $(60 \mu \mathrm{m})$.

\subsection{Characterization}

2.4.1 FTIR. The Fourier transform infrared (FTIR) spectra of polymer films cast from solutions onto potassium bromide

Table 1 Molecular compositions and characterization of $\mathrm{ZnPs}$

\begin{tabular}{|c|c|c|c|c|}
\hline Sample & MMA/EA/2MEA/ZnM ${ }^{a}$ & Viscosity/mPa s & $M_{\mathrm{n}} / \mathrm{g} \mathrm{mol}^{-1}$ & PDI \\
\hline ZnP-1 & $5 / 75 / 10 / 10$ & 180 & $2.3 \times 10^{3}$ & 1.85 \\
\hline $\mathrm{ZnP}-2$ & $5 / 70 / 10 / 15$ & 860 & $2.4 \times 10^{3}$ & 1.96 \\
\hline ZnP-3 & $5 / 65 / 10 / 20$ & 1400 & $2.4 \times 10^{3}$ & 2.18 \\
\hline $\mathrm{ZnP}-4$ & $5 / 60 / 10 / 25$ & 55300 & - & - \\
\hline $\mathrm{ZnP}-5$ & $5 / 75 / 0 / 20$ & 2314 & $2.3 \times 10^{3}$ & 1.88 \\
\hline ZnP-6 & $5 / 70 / 5 / 20$ & 1426 & $2.3 \times 10^{3}$ & 2.06 \\
\hline
\end{tabular}

${ }^{a}$ The weight of $\mathrm{ZnM}$ represents the solid content of zinc acrylate monomer. 
Table 2 Formulation of the ZnP-based self-polishing antifouling paint

\begin{tabular}{|c|c|c|c|c|c|c|c|}
\hline Material $^{a}$ & $\mathrm{ZnP}-1$ to $\mathrm{ZnP}-6$ & $50 \%$ rosin solution & $\mathrm{Cu}_{2} \mathrm{O}$ & CuPT & Pigment & Additives & Solvent \\
\hline $\mathrm{g} / 100 \mathrm{~g}$ paint & 17 & 16 & 40 & 4 & 11 & 12 & 6 \\
\hline
\end{tabular}

plates were recorded using a PerkinElmer Spectrum 100 spectrometer.

2.4.2 GPC. Gel permeation chromatography (GPC) was carried out using a Waters 1515 apparatus (with THF as the eluent at a flow rate of $0.4 \mathrm{~mL} \min ^{-1}$ at $35{ }^{\circ} \mathrm{C}$ ). The instrument was calibrated using polystyrene (PS) standards.

2.4.3 ${ }^{1} \mathrm{H}$ NMR spectroscopy. ${ }^{1} \mathrm{H}$ NMR spectra $(600 \mathrm{MHz})$ were recorded with a Bruker AVANCE instrument using $\mathrm{CDCl}_{3}$ as the solvent at room temperature.

2.4.4 SEM. A scanning electron microscopy (SEM) study was performed to obtain cross-sectional micrographs using a JEOL JSM-6480A microscope equipped with a spectrometer for energy-dispersive X-ray spectroscopy (EDS).

\subsection{Tests of erosion and antifouling properties}

2.5.1 Rotor test. A rotor test was conducted on the selfpolishing coatings to study their polishing effect using a homemade device. Coating solutions were prepared by mixing the synthesized $\mathrm{ZnPs}, 50 \%$ rosin solution, $\mathrm{Cu}_{2} \mathrm{O}$, CuPT, and pigments. The formulation of the self-polishing paint based on the ZnPs is given in Table 2. The sample abbreviations AF-ZnP-1 to AF-ZnP-6 refer to antifouling coatings that were prepared with the ZnP-1 to ZnP-6 resins, respectively. A paint could not be successfully prepared with $\mathrm{ZnP}-4$, which had a high content of bifunctional ZnM, owing to its high viscosity. These samples were coated on tin plates and dried at room temperature for at least a week. Then, the specimens were attached to the inside of a bucket with a rotor to simulate dynamic flushing in artificial seawater at a rotation speed of $550 \mathrm{rpm} .{ }^{23}$ Then, the samples were retrieved and dried before the coating thickness was measured using a thickness gauge. The film thickness was measured at a fixed time, and each sample was measured at nine points to calculate the average film thickness. Then, the specimens were re-immersed in seawater to determine their long-term erosion over the entire test duration.

2.5.2 Raft test. The panels used for the raft test consisted of steel plates, and these panels were sandblasted and coated with an epoxy anti-corrosion paint and a tie coat prior to being coated with the ZnP-based antifouling paints. Each panel $(300 \mathrm{~mm} \times 150 \mathrm{~mm} \times 3 \mathrm{~mm})$ was coated with two paint formulations, and the test area used for each coating had dimensions of $150 \times 150 \mathrm{~mm}$. The coated panels were dried completely at room temperature and lowered into seawater at a depth of 0.2-2.0 $\mathrm{m}$ at two sites (Zhanjiang, Guangdong Province and Qingdao, Shandong Province). After a certain period of time, these panels were removed from the sea, carefully washed with seawater and photographed. Then, the panels were immediately placed back into the sea to continue the test according to the China Standard (GB/T 5370-2007).
Panels were first immersed in the South of China $\left(21^{\circ} 7^{\prime} \mathrm{N}\right.$, $110^{\circ} 14^{\prime} \mathrm{E}$ ) from December 2012 to September 2013. Inspections were performed bimonthly during the hot season, when marine biofouling was severe owing to the wide range of fouling organisms. Steel plates coated with epoxy anti-corrosion paint and a tie coat were used as a blank, and a commercial copperfree antifouling paint (reference-1) from Chugoku Marine Paints, Ltd. (Shanghai, China) was employed as a reference. The tropical and subtropical maritime monsoon climate in this region resulted in an average annual temperature of $23{ }^{\circ} \mathrm{C}$. In addition, coated panels were immersed in the Yellow Sea $\left(36^{\circ} 3^{\prime} \mathrm{N}, 120^{\circ} 25^{\prime} \mathrm{E}\right)$ (NCS Testing Technology Co., Ltd., Qingdao, China), starting in August 2014, when the fouling pressure, as well as the barnacle density, was high. The temperate monsoon climate in this region resulted in an average annual temperature of $12.7{ }^{\circ} \mathrm{C}$. A commercial copper-type antifouling paint (reference-2) from Marine Chemical Research Institute Co., Ltd. (Qingdao, China) and an epoxy tie coat (blank) were used as performance references and negative controls, respectively.

\section{Results and discussion}

\subsection{Chemical compositions of the zinc acrylate copolymers}

In the current study, the ZnPs were synthesized using a two-step method, as shown in Fig. 1. The bifunctional ZnM was first synthesized and then copolymerized with MMA and other comonomers. As shown in Table 1, the molecular composition of the zinc-based random copolymers contained various ratios of the zinc acrylate monomer to the acrylate comonomers. The $M_{\mathrm{n}}$ values of the $\mathrm{ZnPs}$ (ZnP-1 to $\mathrm{ZnP}-3$, ZnP-5 and ZnP-6) were 2300-2400 $\mathrm{g} \mathrm{mol}^{-1}$, which were satisfactorily similar, because a difference in molecular weight can affect the erosion rates of self-polishing coatings.

The chemical structures of the ZnM and ZnPs were analysed by FT-IR spectroscopy. As shown in Fig. 2, the peak observed at $1630-1640 \mathrm{~cm}^{-1}$ was due to the stretching vibrations of $\mathrm{C}=\mathrm{C}$ bonds in the bifunctional ZnM. The intensity of the peaks due to $\mathrm{C}=\mathrm{C}$ bonds in the copolymers decreased significantly in comparison with that for the monomer. In addition, a peak at $1730 \mathrm{~cm}^{-1}$, which corresponded to the carbonyl groups of the ester linkages in the ZnP chains, was observed. All the synthesized ZnPs exhibited prominent absorption peaks at $1605 \mathrm{~cm}^{-1}$, which were due to the asymmetric stretching vibrations of zinc carboxylate groups, and the peaks located at $2900-3000 \mathrm{~cm}^{-1}$ were due to asymmetric and symmetric $\mathrm{C}-\mathrm{H}$ stretching vibrations. ${ }^{18}$

${ }^{1} \mathrm{H}$ NMR spectroscopy was employed to confirm the successful synthesis of the ZnPs. The peaks at 5.5 to $6.5 \mathrm{ppm}$ and $1.92 \mathrm{ppm}$ were assigned to vinyl groups and hydrogen 

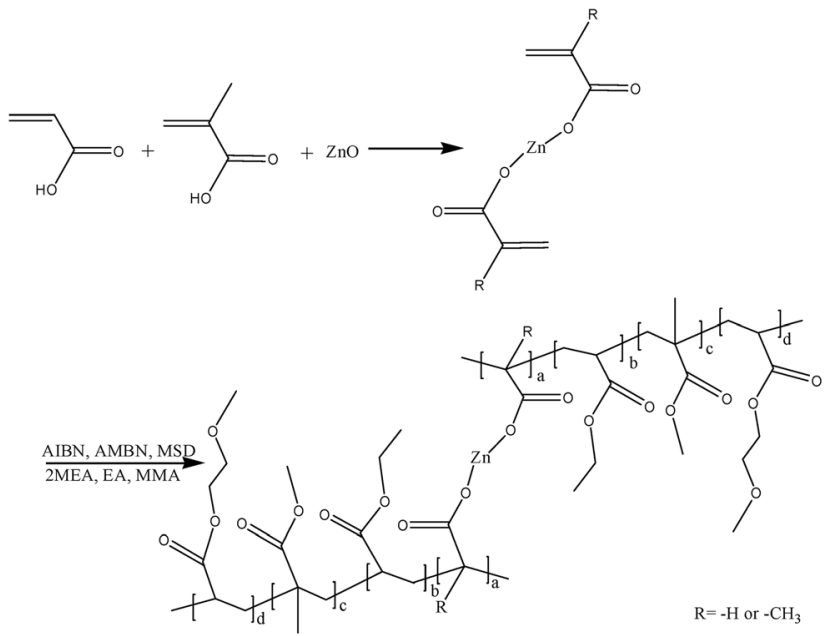

Fig. 1 Synthesis route used for the zinc-based acrylate copolymers (ZnP) containing ZnM.

atoms on the methyl groups of ZnM, respectively (Fig. 1S(a)†). Characteristic peaks corresponding to a double-bond structure at 5.5-6.5 ppm were not observed. However, a peak was observed at 1.6-2.4 ppm for the methylene groups of the polymer (Fig. 1S(b)†). Therefore, the ZnPs were successfully synthesized.

To demonstrate the effect of the synthesized ZnPs on the stability of the paints, the viscosities of the zinc-based coatings are shown in Fig. 3. The viscosities of all the coatings remained at approximately $70 \mathrm{KU}$ for five consecutive months and did not change significantly. This result indicated that the ZnPs did not gel under the influence of the components in the coating, and the prepared coating had high storage stability and good construction properties.

\subsection{Erosion profile of the ZnP-based self-polishing coatings}

To investigate the effect of the monomer composition of the ZnPs on their self-polishing behaviour, the average film thickness of the prepared coatings was determined during the entire duration of the rotor test. In theory, ideal losses of thickness should be observed across the entire surface of each coating for

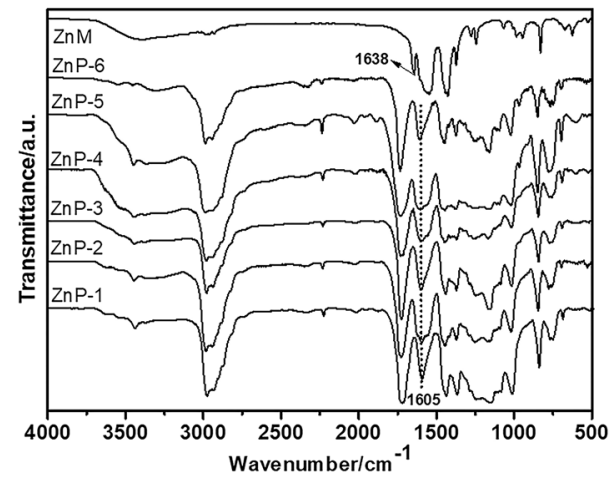

Fig. 2 FT-IR spectra of ZnM and zinc acrylate copolymers.

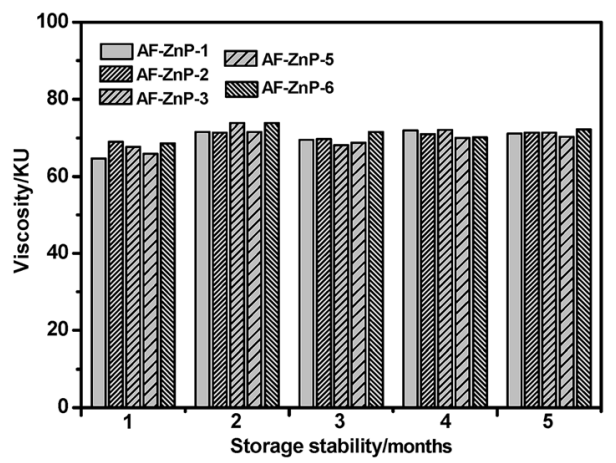

Fig. 3 Storage stability of ZnP-based antifouling coatings.

the hydrolysis of copolymers. Fig. 4 shows the film thickness (a) and relative loss of coating thickness (b) for AF-ZnP-1, AF-ZnP-2, AF-ZnP-3, AF-ZnP-5 and AF-ZnP-6 under the tested immersion conditions. During the first 49 days, all the coatings were only immersed in artificial seawater without dynamic scouring, and the thicknesses of the AF-ZnP-1 and AF-ZnP-2 coatings increased slightly. However, the thickness of the other coatings decreased. During the subsequent erosion test cycles, the thickness of all the coatings increased and then decreased slowly under continuous flushing with seawater. The erosion rate increased as the $\mathrm{ZnM}$ content in the $\mathrm{ZnP}$ increased, as shown in Fig. 4(b). The coating thickness of AF-ZnP-3, AF-ZnP-5 and AF-ZnP-6, which had ZnM contents of $20 \mathrm{wt} \%$, exhibited greater decreases than those of AF-ZnP-1 and AF-ZnP-2, which had ZnM contents of approximately $10 \mathrm{wt} \%$ and $15 \mathrm{wt} \%$, respectively, in the copolymers.

In general, the synthesized ZnPs used for self-polishing coating applications were hydrophobic, which could prevent seawater from penetrating into the paint film. The zinc carboxylate linkage was hydrolytically unstable under slightly alkaline conditions, and its hydrolysis had the result that the ZnPs became to hydrophilic. ${ }^{12}$ The hydrolyzed copolymers could be easily eroded by moving seawater, which led to a decrease in film thickness. However, in comparison with a previous study, ${ }^{18}$ the results indicated that the coating was not polished directly and its thickness decreased linearly and slowly with fluctuations, as expected (Fig. 4). Therefore, the erosion process of the self-polishing coatings was related to both the matrix resin used for solubilization and the formulation of the paint. In this study, a rosin resin was used either as the main controlledrelease resin in the tin-free coatings or to adjust the intrinsic hydrophilicity of the zinc acrylate antifouling systems. ${ }^{24}$ The addition of the hydrophilic rosin resin allowed seawater to penetrate into the paint film and induced swelling of the film, which finally resulted in an increase in the coating thickness. This result indicated that the chain structure and dynamics of the hydrolysable copolymers were greatly affected as water diffused. In addition, the types of pigment and biocide used, as well as mechanical defects due to cracks, may have contributed to the erosion rate of the coatings. ${ }^{15,21}$

Further investigation of the ZnPs was performed using microscopy to determine the depletion profile for the coating 

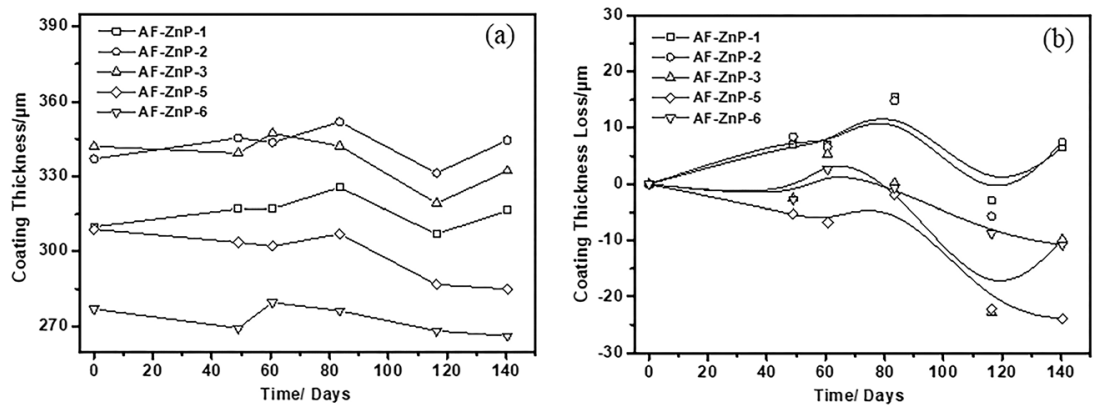

Fig. 4 Film thickness (a) and loss of coating thickness (b) for AF-ZnP-1, AF-ZnP-2, AF-ZnP-3, AF-ZnP-5 and AF-ZnP-6 under the tested immersion conditions.

thickness. Fig. 5 shows a polarizing optical microscopy image (a) and an SEM image (b) of a cross-section of the self-polishing antifouling coating AF-ZnP-5. After immersion, the paint surface was smooth and the cross-section displayed an ambiguous leached layer with two fronts (i.e., a polymer front and a pigment front). The hydrophilic resin and soluble pigments on the surface of the coating dissolved in seawater to leave resin that could not be quickly eroded, which resulted in the appearance of the polymer front. This result indicated that the antifouling coating based on the cross-linked ionomer dissolved smoothly and the $\mathrm{ZnP}$ had a very thin hydrolysis layer, which will result in continuous and effective biocide release rates over time and enhance the antifouling performance.

\subsection{Raft test in natural seawater}

To further investigate their antifouling activity, candidate zincbased coatings containing co-biocides were subjected to a conventional raft immersion test. Visual observations were used to assess the antifouling performance in terms of the adhesion of biomass, including slime, as well as hard and soft fouling organisms. The pressure of fouling varies greatly according to latitude and longitude, as well as environmental conditions, and immersion tests were therefore performed at two natural sites (i.e., in a subtropical area (the South China Sea) and a temperate area (the Yellow Sea)) (Fig. 2S $\dagger$ ). The former immersion site experiences minimal variations in water temperature and light levels, which results in the increased development of encrusting organisms and other macrofoulers.
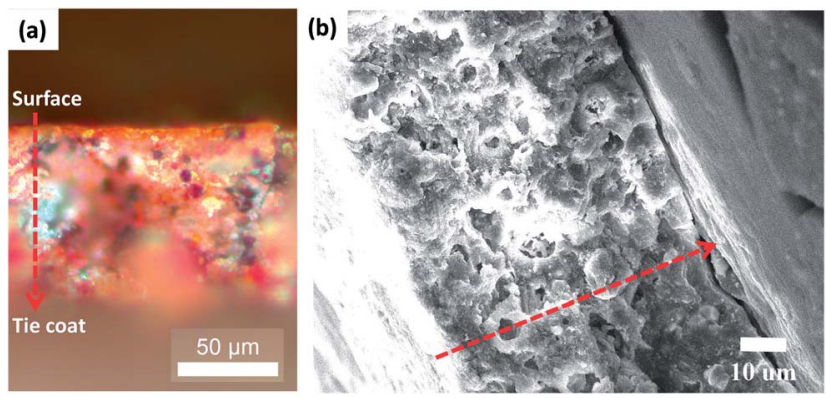

Fig. 5 Polarizing optical microscopy image (a) and SEM picture (b) of a cross-section of AF-ZnP-5 after immersion.
The second immersion site undergoes less fouling development in winter owing to the reduction in daylight hours and seawater temperature, with a main spawning season that extends from spring to late summer. Biofilm, algae and encrusting animals, including bryozoans and tubeworms, are commonly found at both sites. ${ }^{25}$

The activity spectrum of the ZnP-based coatings depends on the biodiversity and fouling pressure. Fig. 6 shows photographic images of coated panels that were immersed in the South China Sea for 9 months. The obverse and reverse sides correspond to the sunlit and dark sides, respectively. The blank panel coated with the epoxy tie coat was nearly completely covered by all types of fouling organisms after a field exposure period of 2 months in the South China Sea. Encrusting species (specifically, barnacles) had settled on $100 \%$ of the coated surface (Fig. $3 \mathrm{~S}(\mathrm{a}) \dagger$ ). When the immersion time in the subtropical area was increased, other macrofouling organisms appeared on this panel, including hydrozoans, bryozoans and so on, which indicated that severe fouling was present in Zhanjiang. In comparison with the blank panel, the antifouling effect of the ZnP-based antifouling coatings was visible. After a field exposure period of 4 months, several plant species and a small number of barnacles settled on most of the coatings, except AF-ZnP-6, which was prepared with ZnP-6. Reference-1, which was a commercial copper-free coating, was nearly completely covered by all types of fouling organisms after immersion for 4 months. Some macrofoulers had fallen off to leave barnacles settled on the coatings after immersion for 6 months. Interestingly, fewer macrofoulers (specifically, barnacles) remained on all the coating surfaces but were mainly replaced by a certain amount of slime after immersion for 9 months in the subtropical area. In general, with the dynamic scouring by seawater, an ion exchange reaction occurred with the $\mathrm{ZnP}$, which led to the erosion of the zinc-based coatings and the gradual release of the antifouling biocides, which confirmed their antifouling performance. However, the growth rate of marine biofoulers varied according to the season, and the breeding period increased the development of encrusting organisms and other macrofoulers in the summer (from April to July). During this period, the polishing rate and biocide release rate could not significantly prevent the adhesion and growth of biofouling. However, the coatings exhibited more beneficial 


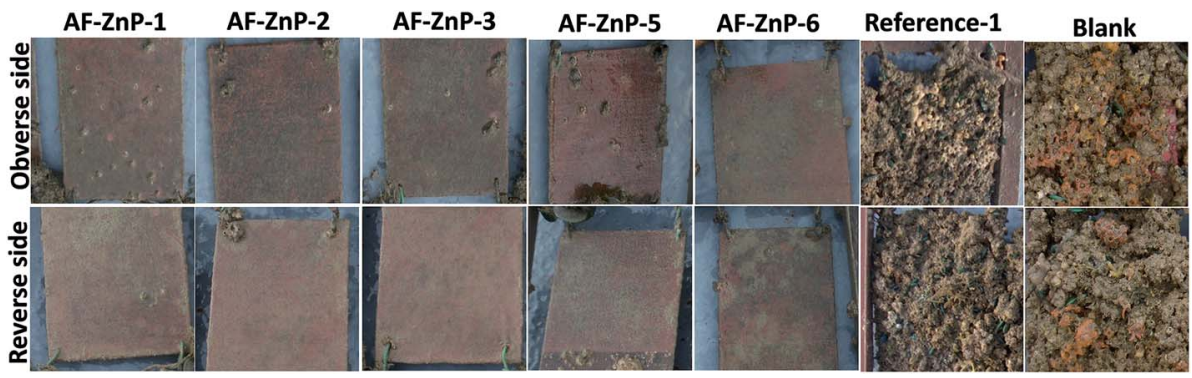

Fig. 6 Images of non-rinsed coated panels that were immersed for 9 months in the South China Sea (Zhanjiang). Reference-1 was a commercial copper-free coating, which was immersed for 6 months.

performance in the autumn. Both the self-polishing effect and the biocides, including $\mathrm{Cu}_{2} \mathrm{O}$ and CuPT, hindered the settlement of barnacles and other encrusting species.

In addition, few fouling organisms settled on the reverse sides in comparison to the obverse sides, as shown in Fig. 6, which demonstrated that the sun played an important role in the species and growth rate of foulers. During a field exposure period of 2-4 months in the South China Sea, most of the tested coatings exhibited a thin cover of fouling, which was dominated by slime films and a small number of juvenile barnacles. After immersion for 9 months, macrofouling was considerably reduced on the ZnP-based self-polishing coatings, but only a small reduction occurred in the covering of slime. In general, the copper-type self-polishing antifouling coatings (i.e., AF-ZnP3, AF-ZnP-5 and AF-ZnP-6), which had ZnM contents of $20 \mathrm{wt} \%$, exhibited better antifouling performance. Therefore, the erosion rate of the ZnP-based coatings was stable, and the coatings prevented the adhesion of different fouling organisms during the biological growth season.

Fig. 7 shows photographic images of self-polishing antifouling coatings based on different ZnPs immersed in the Yellow Sea (Qingdao). The test panels were not rinsed with seawater during the cycle. An assessment of the antifouling performance was carried out in the Yellow Sea for 15 months. When the panels were immersed for 3 months, the entire AF-ZnP-1 sample and half the AF-ZnP-2 and 3 samples were above the sea surface and became grey-white owing to the

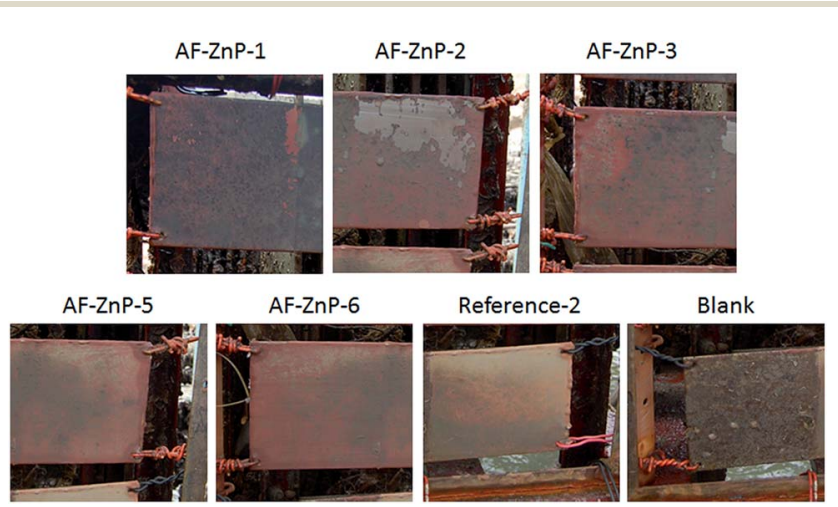

Fig. 7 Images of non-rinsed coated panels immersed for 15 months in the Yellow Sea (Qingdao). decline in sea level in autumn (Fig. $4 \mathrm{~S} \dagger$ ). During a field exposure period of 6 months in the Yellow Sea only the blank panels displayed very little sediment, and after immersion for 9 to 12 months no adherent macrofoulers were observed on the zinc-based candidate coatings. All the coatings exhibited slime and sediment on their surfaces, which were easily removed by wiping, even during the biological growth season. After immersion for 15 months, the panel surfaces with the AF-ZnP-1 and AF-ZnP-2 coatings were still covered with sediment, whereas the amount of sludge on the ZnPs with $\mathrm{ZnM}$ contents of 20 wt\% (AF-ZnP-3, AF-ZnP-5 and AF-ZnP-6) decreased. In comparison with the candidate coatings, the uncoated blank panel was covered by slime and a small number of barnacles.

Nevertheless, all the biocidal coatings in both Zhanjiang and Qingdao were active against the settlement of cyprid larvae and algae owing to their smooth polished surface and toxicity in comparison to the blank panels. The ZnPs with a higher ZnM content exhibited better performance owing to their high erosion rate. In addition, the combination of $\mathrm{Cu}_{2} \mathrm{O}$ and $\mathrm{CuPT}$ exhibited high efficiency against the settlement of algae and the growth of other macrofoulers on the coatings. ${ }^{26}$ Furthermore, the removal of macroorganisms and slime was observed after disturbance by natural seawater in situ on the raft. This result suggested that the erosion of the coatings played two complementary roles on exposure to seawater. Therefore, the results from the field experiments indicated that the coatings with the best performance after immersion for at least 15 months in the Yellow Sea were ZnP-based coatings with ZnM contents of $20 \mathrm{wt} \%$. These ZnP-based coatings exhibited antifouling activities similar to that of a well-known commercial copper-type coating with 5 year antifouling performance (reference-2). It is important to note that the antifouling performance of the selfpolishing coatings was investigated under static conditions. In fact, the coatings are expected to exhibit better performance with the help of shear forces under dynamic conditions. Therefore, the marine field tests demonstrated that the antifouling coatings that were developed have great potential for use in maritime applications.

\subsection{Coating structure and antifouling mechanism}

The results of a previous study indicated that there was a complex relationship between the hydrolysis of the polymer, polishing rate, thickness of the leached layer and antifouling 


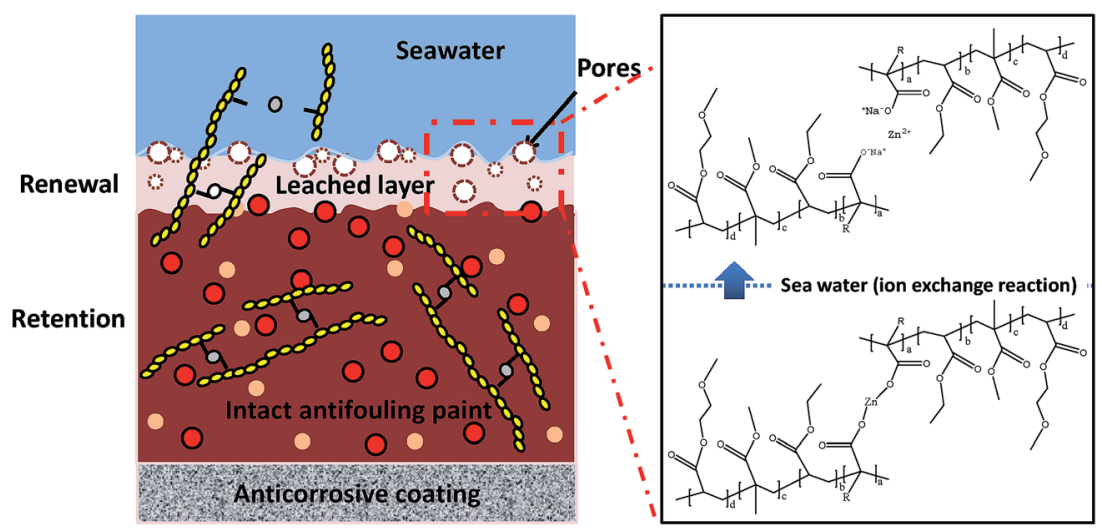

Fig. 8 Schematic of the erosion of the ZnP-based self-polishing antifouling coatings in seawater.

performance. ${ }^{27}$ Fig. 8 shows a schematic illustration of the erosion of the ZnP-based self-polishing antifouling coatings in seawater. Near the interface with seawater, the region appears to be spongy, which corresponds to the hydrated zone caused by the hydrolysis of zinc ester linkages. In moving seawater, this outer layer dissolved into pigment particles and could be easily removed (self-polishing effect). For the self-polishing antifouling coating system, the thickness of the outer layer was heavily influenced by the rate of hydrolysis of the binder, which was important for the overall performance. The specific synthesis of the ZnPs, which differed from that of ordinary acrylic resin, consisted of the formation of a cross-linked ionomer function, as described below. This cross-linked ionomer underwent cleavage of two zinc carboxylate groups from pendant groups on the copolymer backbone, which resulted in the slow and steady erosion of the surface in seawater. This hydrolysis reaction took place throughout the leached layer. Therefore, the thickness of the leached layer reached a steady value. The inner portion remained compact and dense, and degradation did not occur. In the inner portion, biocide molecules did not diffuse because they were in contact with water. ${ }^{21}$

In addition, a rosin resin was used in the antifouling coatings to adjust the intrinsic hydrophobicity and erosion resistance of the coatings to achieve the desired polishing rate. ${ }^{5,28}$ Hydrophilic swelling behaviour increased the movement of the copolymer chains and led to an erosion mechanism that was inhomogeneous across the overall coated surface. In the same way, the water-soluble pigment and biocide components enhanced the permeation of water in the film, which increased the effective contact area of the ZnPs and filler that reacted with seawater. ${ }^{21}$ In this system, the ZnPs simply adjusted the erosion rate of the coatings and did not act as a contact biocide, in contrast to TBTbased coatings. The zinc diacrylate (dimethacrylate) copolymer reacted with sea water, and its subsequent release was a means of controlling the length of the diffusion path of the active substances out of the paint. The continuous release of antifoulants $\left(\mathrm{Cu}_{2} \mathrm{O}\right.$ and $\left.\mathrm{CuPT}\right)$ directly controlled the settlement and growth of fouling organisms. The cooperative effect of the removal of fouling by polishing and the repulsion of organisms via biocidal activity would result in good antifouling performance and ensure that the paint has a long lifetime.

\section{Conclusions}

This study focused on the screening and development of optimized ZnPs as binders for copper-type antifouling coatings. The results of erosion tests performed under dynamic conditions demonstrated the control of the polishing process by coatings based on the copolymers and binders. Field tests further indicated that $\mathrm{ZnP}$-based coatings containing $\mathrm{Cu}_{2} \mathrm{O}$ and $\mathrm{CuPT}$ as cobiocides are promising candidates for inhibiting the settlement of macroorganisms after immersion for at least 15 months in the Yellow Sea and at least 9 months in the subtropical area. The results of this study highlight that the ZnP-based coatings are highly promising candidates for marine antifouling applications.

\section{Conflicts of interest}

There are no conflicts to declare.

\section{Acknowledgements}

This research was supported by the Key Laboratory of Superlight Materials \& Surface Technology (Harbin Engineering University), Ministry of Education, the National Natural Science Foundation of China (51603053), Fundamental Research Funds of the Central Universities (HEUCFM171004), the Application Technology Research and Development Plan of Heilongjiang Province (GX16A008) and the Application Technology Research and Development Projects of Harbin (2015RAQXJ038) and the International S\&T Cooperation Program of China (ISTCP) (No. 2015DFA50050).

\section{References}

1 J. A. Callow and M. E. Callow, Nat. Commun., 2011, 2, 1-10.

2 M. Salta, J. A. Wharton, Y. Blache, K. R. Stokes and J. F. Briand, Environ. Microbiol., 2013, 15, 2879-2893.

3 M. E. Callow and J. A. Callow, Biologist, 2002, 49, 1-5.

4 A. Lindholdt, K. Dam-Johansen, S. M. Olsen, D. M. Yebra and S. Kiil, J. Coat. Technol. Res., 2015, 12, 415-444. 
5 D. M. Yebra, S. Kiil and K. Dam-Johansen, Prog. Org. Coat., 2004, 50, 75-104.

6 M. P. Schultz, Biofouling, 2007, 23, 331-341.

7 M. Candries, M. Atlar, E. Mesbahi and K. Pazouki, Biofouling, 2003, 19, 27-36.

8 H. A. Videla, Int. Biodeterior. Biodegrad., 2002, 49, 259-270.

9 I. B. Beech and J. Sunner, Curr. Opin. Biotechnol., 2004, 15, 181-186.

10 S. Krishnan, C. J. Weinman and C. K. Ober, J. Mater. Chem., 2008, 18, 3405.

11 C. Bressy, C. Hellio, M. N. Nguyen, B. Tanguy, J.-P. Maréchal and A. Margaillan, Prog. Org. Coat., 2014, 77, 665-673.

12 K. Vallée-Rehel, V. Langlois, P. Guérin and A. L. Borgne, J. Environ. Polym. Degrad., 1999, 7, 27-34.

13 I. Omae, Chem. Rev., 2003, 103, 3431-3448.

14 R. Chen, Y. Li, M. Yan, X. Sun, H. Han, J. Li, J. Wang, L. Liu and K. Takahashi, RSC Adv., 2016, 6, 13858-13866.

15 H. Monfared and F. Sharif, Prog. Org. Coat., 2008, 63, 79-86. 16 C. Bressy, A. Margaillan, F. Faÿ, I. Linossier and K. Rehel, Advances in Marine Antifouling Coatings and Technologies, Woodhead Publishing, Cambridge, UK, 2009, pp. 445-491. 17 I. Omae, Appl. Organomet. Chem., 2003, 17, 81-105.
18 B.-W. Kim, T.-W. Kang, H. Park, I. W. Lee, H. H. Chun and N.-J. Jo, Macromol. Res., 2014, 22, 978-982.

19 S.-M. Kim, A. Y. Kim, I. W. Lee, H. Park and D.-H. Hwang, J. Nanosci. Nanotechnol., 2016, 16, 10903-10907.

20 C. Bressy, M. N. Nguyen, B. Tanguy, V. G. Ngo and A. Margaillan, Polym. Degrad. Stab., 2010, 95, 1260-1268.

21 D. M. Yebra, S. Kiil, C. E. Weinell and K. Dam-Johansen, Ind. Eng. Chem. Res., 2006, 45, 1636-1649.

22 Y. Yonehara, H. Yamashita, C. Kawamura and K. Itoh, Prog. Org. Coat., 2001, 42, 150-158.

23 D. Howell and B. Behrends, Biofouling, 2006, 22, 303-315.

24 D. M. Yebra, S. Kiil, K. Dam-Johansen and C. Weinell, Prog. Org. Coat., 2005, 53, 256-275.

25 W. Cao, T. Yan, Z. Li, J. Li and Z. Cheng, Chin. J. Oceanol. Limnol., 2013, 31, 699-711.

26 K. C. Anyaogu, A. V. Fedorov and D. C. Neckers, Langmuir, 2008, 24, 4340-4346.

27 F. Fay, I. Linossier, J. J. Peron, V. Langlois and K. ValleeRehel, Prog. Org. Coat., 2007, 60, 194-206.

28 M. Thouvenin, I. Linossier, O. Sire, J.-J. Péron and K. ValléeRéhel, Macromolecules, 2002, 35, 489-498. 